\title{
Inovação Disruptiva: o Mercado Corporativo de Telefonia e a Tecnologia VoIP
}

\author{
João Armênio Neto \\ UnicenP - Curitiba - PR \\ jarmenio@uol.com.br
}

\begin{abstract}
Resumo. O mercado corporativo de telefonia vem sendo transformado pelo VoIP (Voice over IP), que está mudando o modelo de negócios deste mercado. Trata-se de uma inovação tecnológica significativa que afeta as empresas tradicionais do mercado de telefonia, além de possibilitar a entrada de novos concorrentes. Este artigo faz uma análise do impacto do VoIP no mercado corporativo de telefonia, considerando o momento de turbulência vivenciado a partir da ascensão desta nova tecnologia. O caráter da pesquisa é exploratório e se baseia na análise da história e da situação atual do mercado com o objetivo de comprovar a aplicabilidade da teoria da inovação disruptiva e os seus impactos sobre a telefonia.
\end{abstract}

\section{Introdução}

A telefonia é uma ferramenta de trabalho imprescindível dentro de qualquer empresa. Devido à sua importância, sistemas dedicados foram desenvolvidos para atender à demanda das empresas. Estes sistemas, chamados popularmente de PBX (Private Branch eXchange), foram incorporando funcionalidades, de forma a aumentar a produtividade das pessoas que os utilizam, ao longo do tempo.

Durante mais de vinte anos, o mercado corporativo de telefonia foi dominado por algumas poucas grandes empresas. A base para a telefonia é o serviço de transmissão de voz, que apresenta requisitos específicos como continuidade da transmissão, confiabilidade, disponibilidade, entre outros. Estes requisitos impunham barreiras difíceis de serem superadas por entrantes em potencial, principalmente devido ao alto investimento necessário para o desenvolvimento de know-how e de produtos. Este fato tornava o mercado pouco atrativo para novas empresas, conforme apresentado por Porter (1996), no modelo das cinco forças que atuam contrariamente à lucratividade das empresas.

Durante muito tempo, coexistiram duas redes paralelas e distintas dentro das empresas, uma para voz e outra para dados, característica que ainda hoje predomina, apesar de que já há muito tempo se venha discutindo a convergência das tecnologias e dos serviços por elas proporcionados (MEIRELLES, 1994; MESSERSCHMITT, 1996; HUBAUX et al., 1999).

Já em meados da década de 90, Messerschmitt (1996) preconizava que a integração de todos os tipos de mídia, como voz, áudio, vídeo, animação e dados, em uma mesma rede e em terminais comuns, tornaria difícil a distinção entre telefonia e informática. Neste sentido, já se constatava que os tradicionais setores de telefonia e informática haviam 
sido mudados um pelo outro de forma irreversível. Mas, como restrições a esta integração, Messerschmitt considerava que as disciplinas permaneciam intelectualmente separadas, compartilhando equipamentos e meios de comunicação, mas seguindo agendas distintas e sendo dominadas por culturas diferentes.

Nos últimos anos, uma novidade vem mudando completamente o mercado de telefonia, tanto sob o prisma das operadoras, quanto dos fornecedores de soluções e usuários do mercado corporativo e doméstico deste serviço. Trata-se da tecnologia VoIP (Voice over Internet Protocol), telefonia IP ou telefonia sobre Internet, que nada mais é do que a utilização de redes de dados para a transmissão de voz, o que potencializa a convergência digital.

A introdução desta inovação fez com que as grandes empresas de telefonia, como Alcatel, Avaya, NEC, Nortel e Siemens, tivessem que mudar suas estratégias, passando a suportar esta nova tecnologia. Além disso, novos personagens voltaram sua atenção para a tecnologia VoIP. Entre as empresas entrantes estão aquelas que atuavam no fornecimento de infra-estrutura de comunicação de dados (Cisco e 3Com), em soluções de TI (Microsoft, IBM e Oracle), além de pequenas empresas "aventureiras". Existem até mesmo sistemas em código aberto, dentre os quais o mais conhecido é o Asterisk (GRALLA, 2006).

Tendo sido tecidas essas considerações iniciais, este artigo passa a analisar a tecnologia VoIP como uma inovação disruptiva, como definido por Christensen (1997), dentro do mercado corporativo de telefonia, capaz de proporcionar grandes mudanças no ambiente competitivo e nas competências exigidas dos competidores para nele obterem sucesso.

Primeiramente, será apresentada a abordagem da inovação disruptiva ou tecnologia disruptiva. Em segundo lugar, será analisada a evolução do mercado corporativo de telefonia, e principalmente, da tecnologia VoIP. A seguir, será feita uma análise das avaliações que analistas têm feito sobre os impactos da tecnologia VoIP neste mercado e discutidas as suas expectativas quanto ao futuro. Finalmente, serão apresentadas as considerações finais sobre a forma como a literatura sobre inovação tecnológica ajuda a compreender o que está acontecendo com o mercado de telefonia, após a tão anunciada convergência digital.

\section{Referencial teórico}

Fine (1999) destaca que a visão tradicional da gestão estratégica leva as empresas a buscarem desenvolver vantagem competitiva sustentável. Isso até era possível no tempo em que a velocidade evolutiva dos mercados era menor. $\mathrm{O}$ problema é que o conceito continua sendo aplicado, mas a alta velocidade verificada na evolução de muitos mercados impede que o objetivo de sustentabilidade das estratégias ao longo do tempo seja atingido. Conseguem-se apenas vantagens temporárias.

Christensen et al. (2002) afirmam que a maior parte dos executivos acredita que crescimento significativo e sustentável provém da criação de novos mercados e modos de competição. Porém, a maioria deles produz somente produtos e serviços que atendem à demanda dos clientes já existentes. Os novos produtos e serviços, criados pelas empresas que tradicionalmente disputam um mercado, levam a circunstâncias sustentadoras, em que o resultado se caracteriza por produtos melhores, com preços mais altos e para clientes mais atraentes. Uma vez que é difícil saber como crescer, o 
risco desta abordagem de crescimento é alto (CHRISTENSEN, 2003). Como lembrado por Graeml (2003), “a trajetória das empresas, que garante sucesso em épocas de "calmaria", normalmente as cega para as mudanças, quando estas passam a ocorrer em maior velocidade".

Fine (1999) explica a dificuldade em se obter sustentabilidade estratégica ao apresentar alguns eventos que podem colocar em risco empresas de todas as "espécies"” e "velocidades evolutivas". Em ambientes de alta velocidade evolutiva, a vantagem temporária predomina, ou seja, quanto mais rápida a velocidade evolutiva do setor, mais transiente a vantagem temporária das organizações que o lideram (FINE, 1999).

Para entender melhor os efeitos gerados pela inovação nos mercados, é interessante conhecer a classificação que Christensen (1997) adota para as inovações, a saber: inovação sustentadora (ou tecnologia sustentadora) e inovação disruptiva (ou tecnologia disruptiva).

A inovação sustentadora é aquela que resulta em produtos e serviços que atendem à demanda de clientes existentes em mercados estabelecidos. Inovações sustentadoras ajudam as empresas a aumentar suas margens, vendendo melhores produtos para seus melhores clientes.

A inovação disruptiva, por sua vez, resulta na criação de novos mercados e modelos de negócio, na visão de Christensen (1999). Ela provoca uma ruptura no antigo modelo de negócios e, normalmente, favorece o aparecimento de novos players. A inovação disruptiva usualmente traz para o mercado algo pior em termos de desempenho do que a tecnologia atual, em um primeiro momento. Isto torna seu uso impossível pelos clientes habituais, mas pode permitir que novos clientes, que não se interessam pela sofisticação (e o custo associado) dos produtos disponibilizados pelos fornecedores tradicionais, comecem a comprar.

Gilbert (2003) define três fases no desenvolvimento de inovações disruptivas, a saber: a criação do novo mercado, sua expansão com a redução do mercado estabelecido e, finalmente, a inovação se desenvolve de forma a reduzir a diferença de valor percebido pelos clientes entre o produto tradicional e o disruptivo.

Hart e Christensen (2002) apontam as condições fundamentais para que uma inovação disruptiva tenha sucesso. O produto ou serviço, no início, não deve ser tão bom como os produtos usados pelos clientes nos mercados estabelecidos, para evitar uma reação dos competidores tradicionais em um momento em que o entrante ainda não está fortalecido para suportá-la. Isso faz com que empresas bem sucedidas, pressionadas a apresentar crescimento sustentado e altas margens de lucro, não invistam em inovações disruptivas.

\section{Cronologia do Desenvolvimento da Telefonia - Mercado Corporativo}

Desde que o telefone passou a ser usado no ambiente corporativo como uma ferramenta de trabalho, este mercado vem passando por várias inovações. Dos PBX manuais, passando pelas centrais eletromecânicas ou analógicas, até as centrais digitais com

1 Esse autor se refere a “espécies” em analogia à biologia, em que os indivíduos e espécies mais aptos sobrevivem em um ambiente hostil de competição.

Anais do III Simpósio Brasileiro de Sistemas de Informação. Curitiba, PR, novembro de 2006. 
tecnologia TDM (Time Division Multiplexing), muito se evoluiu, mas sem que o modelo de negócio e os fornecedores tradicionais fossem alterados profundamente (CHAPUIS et al., 1982).

A partir da digitalização da telefonia, torna-se necessário analisar sua evolução em paralelo com a evolução da informática. A evolução tecnológica, em ambas as áreas, vem ocorrendo de forma que uma área se utiliza dos avanços da outra. Messerschmitt (1996) demonstra que esses desenvolvimentos, como a tecnologia comum e a interligação dos computadores em rede, vêm formando a base para a integração entre as telecomunicações e a informática.

No início da década de 80, foi feita a primeira demonstração de uma transmissão de voz sobre a rede de dados (LIU e HAJHAMAD, 2005). Em meados da década de 90, uma empresa israelense chamada VocalTec desenvolveu o primeiro software comercial de VoIP (LIU e HAJHAMAD, 2005). Mas, nesta época, uma conjunção de fatores, como a baixa velocidade de Internet e baixa capacidade de processamento dos processadores, impedia a utilização eficaz da tecnologia.

Somente no final da década de 90, a utilização da tecnologia de forma comercial se tornou possível com a evolução da tecnologia e o desenvolvimento de novas soluções VoIP. Esta evolução se deveu, principalmente, a um aumento na velocidade de transmissão das redes de dados e ao aumento da capacidade de processamento dos microprocessadores e processadores de sinal (DSP - Digital Signal Processor), ainda de acordo com Liu e Hajhamad (2005).

Mas, de acordo com analistas, somente a partir de 2004 verificou-se o ponto de inflexão na procura por PBX IP (MACHOWINSKI, 2005). Neste ano, a procura por sistemas IP passou a substituir a procura por linhas TDM.

Vários analistas citam um artigo do Gartner Group denominado The IP-PBX is a Potential Architectural 'Dead End', que afirma que, "até 2009, os planos da telefonia corporativa vão migrar de um PBX IP para um modelo de aplicação distribuído de voz" (VANDERMATE, 2006; KASS, 2006). Schulzrinne (2004) alega que o VoIP pode ser visto de duas formas diferentes. Uns o analisam como mais uma evolução similar a outras que já aconteceram no passado na telefonia. Outros, acreditam que o VoIP nada mais é do que mais uma aplicação da Internet que permite a interligação com os sistemas legados de voz.

\section{Metodologia}

Este estudo tem como objetivo explorar o efeito de uma nova tecnologia disruptiva, como o VoIP, sobre o mercado corporativo de telefonia, que foi, nas últimas décadas, dominado por um número restrito de participantes. Foram coletadas as análises feitas por analistas e empresas de consultoria do mercado, dentre os quais merecem destaque a Forrester Research, Frost \& Sullivan, Infonetics Research, além de revistas e sites especializados como: VoIP Planet, Convergência Digital, iIWire, Computer World, VoIP News, Business Communications Review. A partir dessas análises, o autor do presente artigo procurou identificar a situação das empresas tradicionais e também das empresas entrantes neste mercado, à luz da teoria apresentada na sessão anterior. 


\section{Análise do Impacto no Mercado}

Segundo o instituto Frost\&Sullivan, em Rao et al. (2006), o mercado global de telefonia corporativa foi estimado em 7,59 bilhões de dólares em 2005, apresentando um crescimento de aproximadamente $10 \%$ em relação ao ano anterior.

O instituto Infonetics (2006) estimou, para 2005, um volume de 8,1 bilhões de dólares, um aumento de $12 \%$ em relação a 2004. Estima também um crescimento de $43 \%$ até 2009. A estimativa da Infonetics é que, neste período, o faturamento com IP PBX cresça $82 \%$, enquanto o faturamento com PBX TDM deve cair $88 \%$.

O instituto Forrester verificou que, em 2005, 12\% das empresas estavam implementando VoIP e que, em 2006, a proporção passou para 40\% (PIERCE, 2006). Dentre as empresas americanas, $51 \%$ esperam ter migrado seus sistemas de telefonia completamente para VoIP até 2010.

O mercado corporativo de telefonia era dominado por um número restrito de empresas, onde no mercado de médio e grande porte, apenas algumas grandes empresas se destacavam, como Alcatel, Avaya (separada da Lucent em 2000), NEC, Nortel e Siemens (SULKIN, 2006). Em relação ao VoIP, estas empresas traçaram uma estratégia de migração da tecnologia TDM para a tecnologia VoIP. (SULKIN, 2006; MACHOWINSKI, 2006).

Entre os entrantes, destacam-se Cisco, 3Com, Microsoft, IBM e Oracle. A Cisco e a 3Com, tradicionais fornecedores de infraestrutura de dados, iniciaram sua atuação através da aquisição de pequenas empresas em 1998. A Cisco surpreende os principais concorrentes pelo crescimento acima do esperado (SULKIN, 2006). A Microsoft, tradicional fornecedora de infraestrutura de TI tem parceria com quase todos os fornecedores do setor, mas causa apreensão por se apresentar como um concorrente em potencial (HETTICK, 2006). Também começam entrar neste mercado, IBM e Oracle, com soluções que suportam VoIP (RAO, 2006).

Seguindo o caminho do Linux, soluções de software em código aberto para VoIP tem potencial para causar um impacto considerável neste mercado. Atualmente, as soluções de software livre são apropriadas para pequenas e médias empresas, mas o potencial de crescimento existe também no mercado das grandes empresas (GRALLA, 2006).

O serviço de Hosted IP PBX passa a ser oferecido não somente pelas tradicionais operadoras de telefonia como também por provedores de serviço (LEWIS, 2006).

Já os integradores têm muito a ganhar com a migração da agregação de valor na cadeia, da venda e locação de HW, para a venda e integração de software. Além disso, as soluções em código aberto aumentam o seu poder de atuação, diminuindo sua dependência dos grandes fornecedores (GRALLA, 2006).

Como se pode notar, o mercado de telefonia, que movimenta aproximadamente 8 bilhões de dólares por ano e que se concentrava em 5 grandes empresas, passou a ser disputado por pelo menos outros 5 "pesos-pesados" da área de TI e mais alguns players entrantes no mercado. Além disso, todo o modelo de negócios foi modificado, e ainda tem potencial para mais mudanças, uma vez o modelo atual ainda não é definitivo. 


\section{Análise sob o prisma da teoria das inovações disruptivas}

O mercado corporativo de telefonia sofreu inúmeras inovações, que resultaram em produtos melhores, a preços mais altos e para clientes mais exigentes, o que é típico da inovação sustentadora. Além do que, por muitos anos, não criou condições favoráveis para a entrada de outros players no mercado.

A tecnologia VoIP, por sua vez, pode ser classificada como uma inovação disruptiva (LIU et al., 2005), já que essa nova tecnologia apresenta, em sua evolução, características marcantes que coincidem com aquelas citadas por Christensen (1997) para definir inovações disruptivas:

- ela começou como uma tecnologia com menor qualidade do que a tecnologia estabelecida. No início de sua trajetória, o VoIP apresentava problemas de qualidade no sinal de voz, confiabilidade, disponibilidade e segurança;

- não se pode afirmar que VoIP seja uma tecnologia mais simples do que a TDM, mas, por outro lado, simplifica a operação, pois elimina a necessidade de se manter duas redes paralelas independentes, uma para voz e outra para dados;

- possibilitou a entrada de novos players no setor, como: Cisco, 3Com, Microsoft, IBM, Oracle, Open Source VoIp, Hosted IP PBX entre outros.

- mudou o modelo de negócios, migrando o valor na cadeia de valores da venda e arrendamento de HW para as licenças de SW e os serviços de integração.

A forma como evoluiu a telefonia tradicional, focando nas inovações sustentadoras e tentando evitar a canibalização pelo VoIP, também é perfeitamente explicada pelo Modelo de Tecnologia Disruptiva apresentado por Christensen (1999):

- A tecnologia sustentadora havia se desenvolvido até um ponto em que, em grande parte, excedia as necessidades dos clientes;

- A tecnologia disruptiva não foi diretamente adotada pelos players tradicionais porque, inicialmente, era pior do que a tecnologia sustentadora. Mas, com o passar do tempo, evoluiu de forma a apresentar mais vantagens do que desvantagens para o cliente médio.

No que diz respeito à estratégia das empresas envolvidas, nota-se que as que já estavam no mercado tentaram manter suas posições, oferecendo produtos compatíveis com a tecnologia tradicional, mas com a possibilidade de migração para a nova tecnologia. Por outro lado, as empresas entrantes no mercado de telefonia oferecem uma visão de ruptura com a tecnologia antiga.

\section{Conclusão}

A revolução causada pelo VoIP ainda está apenas no início. Muitos ajustes ainda ocorrerão até que esta tecnologia possa ser considerada madura. Temas como segurança, disponibilidade, confiabilidade, entre outros, ainda precisam ser melhor desenvolvidos.

A migração de um mercado baseado em hardware dedicado (telefonia TDM), passando por sistemas híbridos (TDM com acesso a telefonia IP), até os atuais sistemas puramente IPs, parece não ter atingido o status final. Acredita-se que o destino final 
possa ser a transformação do serviço de voz em uma mera aplicação dentro dos sistemas de Tecnologia de Informação, como acontece hoje com o e-mail.

A convergência digital, de certa forma potencializada pelo VoIP, abre um leque de outras mudanças, como por exemplo o conceito de Unified Communications. Existem assim, dentro deste contexto dinâmico em que este setor está inserido, muitas possibilidades para a realização de estudos acadêmicos. Não foi avaliado neste artigo, por exemplo, o efeito causado pelo VoIP na verticalização ou horizontalização do mercado de telefonia, além das questões referentes a aprisionamento e guerra de padrões. A estratégia adotada por cada uma das empresas envolvidas, e seus respectivos resultados, poderiam ser objeto de diversos estudos de casos, que poderiam ajudar a esclarecer ainda melhor as perspectivas deste mercado.

Não é fácil antecipar até onde se vai chegar. Em muitos casos, percebe-se que, se por um lado, os participantes tradicionais de um mercado ficam cegos pela sua trajetória de sucesso, os próprios introdutores de inovações disruptivas não têm, no início, a noção clara do potencial das mudanças por eles propostas. Ainda assim, é reconfortante encontrar teorias que ajudem a explicar melhor a prática, possibilitando ao menos a tentativa de se ver mais longe!

\section{Referências}

CHAPUIS, R.J.; JOEL, A.E. 100 Years of Telephone Switching (1878-1978: Part 1: Manual and Electromechanical Switching). Amsterdam: Elsevier Science Ltd, 1982.

CHRISTENSEN, C. M. The Innovator's Solution: O Crescimento pela Inovação. Rio de Janeiro: Campus, 2003.

CHRISTENSEN, C. M. The Innovator’s Dilemma. Executive Forum Series, 1999.

CHRISTENSEN, C. M. The Innovator's Dilemma: When New Technologies Cause Great Firms to Fail. Boston: Harvard Business School Press, 1997.

CHRISTENSEN, C. M.; JOHNSON, W.J.; RIGBY, D.K.. Foundations for Growth: How To Identify and Build Disruptive New Businesses. MIT Sloan Management Review, Boston, v.43, n.3, SPRING 2002.

FINE, C. Mercados em Evolução Contínua. Rio de Janeiro: Campus, 1999.

GILBERT, C. The Disruption Opportunity. MIT Sloan Management Review, Boston, v.44, n.4, SUMMER 2003.

GRAEML, Alexandre R. Sistemas de Informação: o alinhamento da TI com a estratégia competitiva. São Paulo: Atlas, 2003.

GRALLA, P. Throw away your PBX: Why Asterisk may be the VoIP future of your network, Computer World, Setembro de 2006. Disponível em: http://www.computerworld.com/action/article.do?command=viewArticleBasic\&taxono myName=voip\&articleId=9003067\&taxonomyId=81. Acesso em 18/09/2006.

HETTICK, L. Microsoft: IP-PBX Partner or Competitor?. Business Communication Review. v.33, n.7, p. 24. Jul. 2006

HUBAUX, J.P.; GBAGUIDI, C.; KOPPENHOEFER, S.; LE BOUDEC, J.Y. The impact of the Internet on telecommunication architectures. Computer Networks, 1999. 
INFONETICS RESEARCH. Enterprise VoIP adoption in North America will more than double in 2010. Boston., May 10, 2006.

KASS, D. H. Enterprise VoIP adoption fuels sudden IP PBX boom, America's Network, 2006.

LEWIS, C. Voice over IP special report: Voice gets louder ComputerWeekly.com Fevereiro de 2006. Disponível em: http://www.computerweekly.com/Articles/2006/02/14/214144/Voice+over+IP+special+ report+Voice+gets+louder.htm. Acesso em: 19/09/2006.

LIU, J., \& HAJHAMAD B. The Business of VoIP. MIT Sloan School of Management, May 2005.

MACHOWINSKI, M. Enterprise VoIP: To adopt or not to adopt? Infonetics Research, 2005.

MEIRELLES, F. S. Informática, novas aplicações com microcomputadores. São Paulo: Makron Books, 1994.

MESSERSCHMITT, D. G. The Convergence of Telecommunications and Computing: What are the implications today? Proceedings of IEEE, 1996.

PIERCE, L. The State of Enterprise Telecom and Network Adoption. Forrester Research, 2006.

PORTER, M. E. Estratégia Competitiva. São Paulo: Campus, 1986.

RAO, K., Telephony Joins the Ranks of a Broader Enterprise Application Portfolio. Frost \& Sullivan Market Insight, Oct. 2006.

RAO, K., CRISPIN, K., BANERJEE, S., \& GAITONDE P. Siemens Communications to Shed its Enterprise Solutions Group - Could this be the beginning of some mega mergers in the enterprise telephony space? Frost \& Sullivan, 2006.

SCHULZRINNE, H. INTERNET telephony or Internet TELEPHONY? Columbia University, 2004.

SULKIN, A. IP-Telephony System Market Chugging Along. Business Communication Review. v.36, n.1, Jan. 2006. 